\title{
Operational Calibration: Debugging Confidence Errors for DNNs in the Field
}

\author{
Zenan Li \\ State Key Lab of Novel Software \\ Technology, Nanjing University \\ Nanjing, China \\ lizenan@smail.nju.edu.cn \\ Jingwei $\mathrm{Xu}$ \\ State Key Lab of Novel Software \\ Technology, Nanjing University \\ Nanjing, China \\ jingweix@nju.edu.cn
}

\author{
Xiaoxing $\mathrm{Ma}^{*}$ \\ State Key Lab of Novel Software \\ Technology, Nanjing University \\ Nanjing, China \\ xxm@nju.edu.cn \\ Chun Cao \\ State Key Lab of Novel Software \\ Technology, Nanjing University \\ Nanjing, China \\ caochun@nju.edu.cn
}

\author{
Chang Xu \\ State Key Lab of Novel Software \\ Technology, Nanjing University \\ Nanjing, China \\ changxu@nju.edu.cn \\ Jian Lü \\ State Key Lab of Novel Software \\ Technology, Nanjing University \\ Nanjing, China \\ lj@nju.edu.cn
}

\begin{abstract}
Trained DNN models are increasingly adopted as integral parts of software systems, but they often perform deficiently in the field. A particularly damaging problem is that DNN models often give false predictions with high confidence, due to the unavoidable slight divergences between operation data and training data. To minimize the loss caused by inaccurate confidence, operational calibration, i.e., calibrating the confidence function of a DNN classifier against its operation domain, becomes a necessary debugging step in the engineering of the whole system.

Operational calibration is difficult considering the limited budget of labeling operation data and the weak interpretability of DNN models. We propose a Bayesian approach to operational calibration that gradually corrects the confidence given by the model under calibration with a small number of labeled operation data deliberately selected from a larger set of unlabeled operation data. The approach is made effective and efficient by leveraging the locality of the learned representation of the DNN model and modeling the calibration as Gaussian Process Regression. Comprehensive experiments with various practical datasets and DNN models show that it significantly outperformed alternative methods, and in some difficult tasks it eliminated about $71 \%$ to $97 \%$ high-confidence $(>0.9)$ errors with only about $10 \%$ of the minimal amount of labeled operation data needed for practical learning techniques to barely work.
\end{abstract}

\section{CCS CONCEPTS}

- Software and its engineering $\rightarrow$ Software testing and debugging; • Computing methodologies $\rightarrow$ Neural networks.

${ }^{*}$ Corresponding author.

This work is licensed under a Creative Commons Attribution-NonCommercial International 4.0 License.

ESEC/FSE '20, November 8-13, 2020, Virtual Event, USA

(C) 2020 Copyright held by the owner/author(s).

ACM ISBN 978-1-4503-7043-1/20/11.

https://doi.org/10.1145/3368089.3409696

\section{KEYWORDS}

Operational Calibration, Deep Neural Networks, Gaussian Process

ACM Reference Format:

Zenan Li, Xiaoxing Ma, Chang Xu, Jingwei Xu, Chun Cao, and Jian Lü. 2020. Operational Calibration: Debugging Confidence Errors for DNNs in the Field. In Proceedings of the 28th ACM Foint European Software Engineering Conference and Symposium on the Foundations of Software Engineering (ESEC/FSE '20), November 8-13, 2020, Virtual Event, USA. ACM, New York, NY, USA, 13 pages. https://doi.org/10.1145/3368089.3409696

To know what you know and what you do not know, that is true knowledge.

Confucius. $551-479 \mathrm{BC}$.

\section{INTRODUCTION}

Deep learning (DL) has achieved human-level or even better performance in some difficult tasks, such as image classification and speech recognition [13, 23]. Deep Neural Network (DNN) models are increasingly adopted in high-stakes application scenarios such as medical diagnostics [36] and self-driven cars [3]. However, it is not uncommon that DNN models perform poorly in practice [47]. The interest in the quality assurance for DNN models as integral parts of software systems is surging in the community of software engineering [19, 28, 43, 56, 67, 68].

A particular problem of using a previously well-trained DNN model in an operation domain is that the model may not only make more-than-expected mistakes in its predictions, but also give erroneous confidence values for these predictions. The latter issue is particularly problematic for decision making, because if the confidence values were accurate, the model would be at least partially usable by accepting only high-confidence predictions. It needs to be emphasized that erroneous predictions with high confidence are especially damaging because users will take high stakes in them. For example, an over-confident benign prediction for a pathology image could mislead a doctor into overlooking a malignant tumor.

The problem comes from the almost inevitable divergences between the original data on which a model is trained and the actual data in its operation domain, which is often called domain shift or dataset shift [34] in the machine learning literature. It can be 
difficult and go beyond the stretch of usual machine learning tricks such as fine-tuning and transfer learning [38, 63], because of two practical restrictions often encountered. First, the training data of a third-party DNN model are often unavailable due to privacy and proprietary limitations [20,54,69]. Second, one can only use a small number of labeled operation data because it could be very expensive to label the data collected in the field. For example, in an AI-assisted clinical medicine scenario, surgical biopsies may have to be involved in the labeling of radiology images.

We consider operational calibration that corrects the error in the confidence provided by a DNN model for its prediction on each input in a given operation domain. It does not change the predictions themselves but tells when the model works well and when not. As the quantification of the intrinsic uncertainty in the predictions made by a model, confidence values are integral parts of the model's outputs. So operational calibration can be viewed as a kind of debugging activity that identifies and fixes errors in these parts of model outputs. It improves the model's quality of service in the field with more accurate confidence for better decision making. As a quality assurance activity, operational calibration shall be carried out during the deployment of a previously trained DNN model in a new operation domain. One can also incorporate it into the system and excise it from time to time to adapt the model to the evolving data distribution in the operation domain.

It is natural to model operational calibration as a case of nonparametric Bayesian Inference and solve it with Gaussian Process Regression [46]. We take the original confidence of a DNN model as the prior, and gradually calibrate the confidence with the evidence collected by selecting and labeling operation data. The key insight into effective and efficient regression comes from two observations: First, the DNN model, although suffering from the domain shift, can be used as a feature extractor with which unlabeled operation data can be nicely clustered $[55,72]$. In each cluster, the prediction correctness of an example is correlated with another one. The correlation can be effectively estimated with the distance of the two examples in the feature space. Second, Gaussian Process is able to quantify the uncertainty after each step, which can be used to guide the selection of operation data to label efficiently.

Systematic empirical evaluations showed that the approach was promising. It outperformed existing calibration methods in both efficacy and efficiency in all settings we tested. In some difficult tasks, it eliminated about $71 \%$ to $97 \%$ high-confidence errors with only about $10 \%$ of the minimal amount of labeled operation data needed for practical learning techniques to barely work.

In summary, the contributions of this paper are:

- Examining quality assurance for DNN models used as software components, and raising the problem of operational calibration as debugging for confidence errors of DNNs in the field.

- Proposing a Gaussian Process-based approach to operational calibration, which leverages the representation learned by the DNN model under calibration and the locality of confidence errors in this representation.

- Evaluating the approach systematically. Experiments with various datasets and models confirmed the general efficacy and efficiency of our approach.
The rest of this paper is organized as follows. We first discuss the general need for operational quality assurance for DNNs in Section 2, and then define the the problem of operational calibration in Section 3. We detail our approach to operational calibration in Section 4 and evaluate it empirically in Section 5. We overview related work and highlight their differences from ours in Section 6 before concluding the paper with Section 7 .

\section{QUALITY ASSURANCE FOR DNN MODELS USED AS SOFTWARE ARTIFACTS}

Well trained DNN models can provide marvelous capabilities, but unfortunately their failures in applications are also very common [47]. When using a trained model as an integral part of a high-stakes software system, it is crucial to know quantitatively how well the model will work and to adapt it to the application conditions. The quality assurance combining the viewpoints from software engineering and machine learning is needed, but largely missing. In what follows, we first discuss the non-conventional requirements for such quality assurance, and then give an application scenario to highlight the software engineering concerns.

Deep learning is intrinsically inductive $[13,70]$. However, conventional software engineering is mostly deductive, as evidenced by its fundamental principle of specification-implementation consistency. A specification defines the assumptions and guarantees of a software artifact. The artifact is expected to meet its guarantees whenever its assumptions are satisfied. Thus explicit specifications make software artifacts more or less domain independent. However, statistical machine learning does not provide such kind of specifications. Essentially it tries to induce a model from its training data, which is intended to be general so that the model can give predictions on previously unseen inputs. Unfortunately the scope of generalization is unspecified. As a result, a major problem comes from the divergence between the domain where the model was originally trained and the domain where it actually operates.

So the first requirement for the quality assurance of a DNN model is to be operational, i.e., to focus on the concrete domain where the model actually operates. Logically speaking, the quality of a trained DNN model will be pointless without considering its operation domain. In practice, the performance of a model may drop significantly with domain shift [26]. On the other hand, focusing on the operation domain also relieves the DNN model from depending on its original training data. Apart from practical concerns such as protecting the privacy and property of the training data, decoupling a model from its training data and process will also be helpful for (re)using it as a commercial off-the-shelf (COTS) software product [69]. This viewpoint from software engineering is in contrasting to machine learning techniques dealing with domain shift such as transfer learning or domain adaptation that heavily rely on the original training data and hyperparameters [38, 55, 61]. They need original training data because they try to generalize the scope of the model to include the new operation domain.

The second requirement is to embrace the uncertainty that is intrinsic in DNN models. A defect, or a "bug", of a software artifact is a case that it does not deliver its promise. Different from conventional software artifacts, a DNN model never promises to be certainly correct on any given input, and thus individual incorrect 
predictions per se should not be regarded as bugs, but to some extent features [17]. Nevertheless, the model statistically quantifies the uncertainty of their predictions. Collectively, it is measured with metrics such as accuracy or precision. Individually, it is stated by the confidence value about the prediction on each given input. These qualifications of uncertainty, as well as the predictions a model made, should be subject to quality assurance. For example, given a DNN model and its operation domain, operational testing [26] examines to what degree the model's overall accuracy is degraded by the domain shift. Furthermore, operational calibration, which is the topic of the current paper, identifies and fixes the misspecified confidence values on individual inputs.

Finally, operational quality assurance should prioritize the saving of human efforts, which include the cost of collecting, and especially labeling, the data in the operation domain. The labeling of operation data often involves physical interactions, such as surgical biopsies and destructive testings, and thus can be expensive and time-consuming. Note that, as exemplified by the EU GDPR [8], there are increasing concerns about the privacy and property rights in the data used to train DNN models. So when adopting a DNN model trained by a third party, one should not assume the availability of its original training data [20, 54, 69]. Without the access to the original training data, re-training or fine-tuning a DNN model to an operation domain can be unaffordable because it typically requires a large amount of labeled examples to work. Quality assurance activities often have to work under a much tighter budget for labeling data.

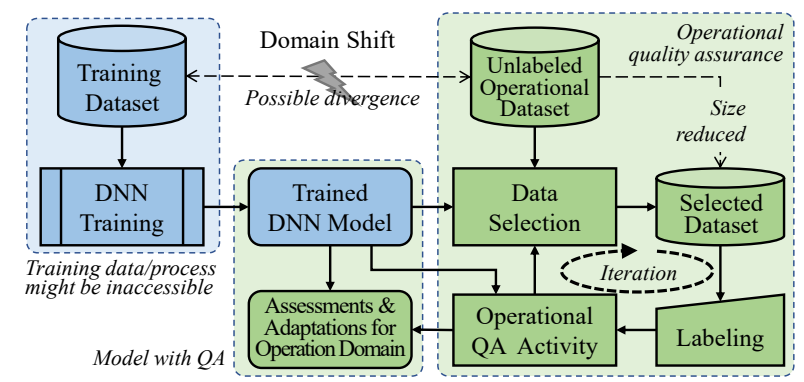

Figure 1: Operational quality assurance

Figure 1 depicts the overall idea for operational quality assurance, which generalizes the process of operational testing proposed in [26]. A DNN model, which is trained by a third party with the data from the origin domain, is to be deployed in an operation domain. It needs to be evaluated, and possibly adapted, with the data from the current operation domain. To reduce the effort of labeling, data selection can be incorporated in the procedure with the guidance of the information generated by the DNN model and the quality assurance activity. Only the DNN models that pass the assessments and are possibly equipped with the adaptations will be put into operation.

For example, consider a scenario that a hospital decides to equip its radiology department with automated medical image analysis enabled by Deep Learning [36]. While the system may involve many functionalities such as clinical workflow management, computeraided diagnosis, and computer-assisted reporting, the key component is a DNN model or an ensemble of DNN models acting as a radiologist to classify images [31]. It is too expensive and technically demanding for the hospital to collect enough high quality data and train the model in-house. So the hospital purchases the model from a third-party provider, and uses it as a COTS software component. However, the training conditions of the model are likely to be different from the operation conditions, and the model performance reported by the provider is unreliable due to potential issues such as data mismatch, selection bias, and non-stationary environments $[59,66]$. To assure the system's quality of service, the model must be tested and calibrated against the current operation domain. The hospital first assesses the real accuracy of the model through operational testing [26], and decides to adopt it or not accordingly. Once the model is adopted and deployed, operational calibration steps in to adapt the model to the current operation domain, by fixing the errors in the confidence values and avoiding high-confidence false predictions.

As a software quality assurance task, operational calibration tries to achieve best efficacy with a limited budget. The cost here, however, is mainly spent on the labeling of operation data. For sophisticated DNN models used for medical imaging classification such as Inception-V3, ResNet-50 and DenseNet-121 [27, 66], even thousands of labeled data could be too less for model retraining or fine-tuning to work properly (c.f. Figure 3). Nevertheless, as will be shown later, a deliberately designed calibration method can identify and fix most of the erroneous high confidence values associated to false predictions with only tens to small hundreds of labeled data (c.f. Figure 6). A model well calibrated for its operation domain, although still suffering from some loss in prediction accuracy, becomes more reliable in that broken promises are far less likely.

\section{OPERATIONAL CALIBRATION PROBLEM}

Now we focus on the problem of operational calibration. We first briefly introduce DNN classifiers and their prediction confidence to pave the way for the formal definition of the problem.

\subsection{DNN Classifier and Prediction Confidence}

A deep neural network classifier contains multiple hidden layers between its input and output layers. A popular understanding [13] of the role of these hidden layers is that they progressively extract abstract features (e.g., a wheel, human skin, etc.) from a highdimensional low-level input (e.g., the pixels of an image). These features provide a relatively low-dimensional high-level representation $\boldsymbol{z}$ for the input $\boldsymbol{x}$, which makes the classification much easier, e.g., the image is more likely to be a car if wheels are present.

What a DNN classifier tries to learn from the training data is a posterior probability distribution, denoted as $p(y \mid \boldsymbol{x})$ [2]. For a $K$-classification problem, the distribution can be written as $p_{i}(\boldsymbol{x})=p(y=i \mid \boldsymbol{x})$, where $i=1,2, \ldots, K$. For each input $\boldsymbol{x}$ whose representation is $z$, the output layer first computes the nonnormalized prediction $\boldsymbol{h}=\boldsymbol{W}^{\top} \boldsymbol{z}+\boldsymbol{b}$, whose element $h_{i}$ is often called the logit for the $i$-th class. The classifier then normalizes $\boldsymbol{h}$ 
with a softmax function to approximate the posterior probabilities

$$
\hat{p}_{i}(\boldsymbol{x})=\operatorname{softmax}(\boldsymbol{h})_{i}=\frac{e^{h_{i}}}{\sum_{j=1}^{K} e^{h_{j}}}, \quad i=1, \ldots, K .
$$

Finally, to classify $\boldsymbol{x}$, one just chooses the the category corresponding to the maximum posterior probability, i.e.,

$$
\hat{y}(\boldsymbol{x})=\underset{i}{\arg \max } \hat{p}_{i}(\boldsymbol{x}) .
$$

Obviously, this prediction is intrinsically uncertain. The confidence for this prediction, which quantifies the likelihood of correctness, can be naturally measured as the estimated posterior class probability

$$
\hat{c}(\boldsymbol{x})=\hat{p_{i}}(\boldsymbol{x}), \quad i=\hat{y}(\boldsymbol{x}) .
$$

Confidence takes an important role in decision-making. For example, if the loss due to an incorrect prediction is four times of the gain of a correct prediction, one should not invest on predictions with confidence less than 0.8 .

Modern DNN classifiers are often inaccurate in confidence [57], because they overfit to the surrogate loss used in training [14, 58]. Simply put, they are over optimized toward the accuracy of classification, but not the accuracy of estimation for posterior probabilities. To avoid the potential loss caused by inaccurate confidence, confdence calibration can be employed in the learning process [10, 14, 58]. Early calibration methods such as isotonic regression [65], histogram binning [65], and Platt scaling [45] simply train a regression model taking the uncalibrated confidence as input with the validation dataset. More flexible methods, e.g., Temperature Scaling [16], find a function $R$ to correct the logit $\boldsymbol{h}$ such that

$$
\hat{c}(\boldsymbol{x})=\hat{p_{i}}(\boldsymbol{x})=\operatorname{softmax}(R(\boldsymbol{h}))_{i}, \quad i=\hat{y}(\boldsymbol{x})
$$

matches the real posterior probability $p_{i}(\boldsymbol{x})$. Notice that, in this setting the inaccuracy of confidence is viewed as a kind of systematic error or bias, not associated with particular inputs or domains. That is, the calibration does not distinguish between different inputs with the same uncalibrated confidence. .

\subsection{Operational Confidence Calibration}

Given a domain where a previously trained DNN model is deployed, operational calibration identifies and fixes the model's errors in the confidence of predictions on individual inputs in the domain. Operational calibration is conservative in that it does not change the predictions made by the model, but tries to give accurate estimations on the likelihood of the predictions being correct. With this information, a DNN model will be useful even though its prediction accuracy is severely affected by the domain shift. One may take only its predictions on inputs with high confidence, but switch to other models or other backup measures if unconfident.

To quantify the accuracy of the confidence of a DNN model on a dataset $D=\left\{\left(\boldsymbol{x}_{i}, y_{i}\right), i=1, \ldots, N\right\}$, one can use the Brier score (BS) [4], which is actually the mean squared error of the estimation:

$$
B S(D)=\frac{1}{N} \sum_{i=1}^{N}\left(\mathbb{I}\left(\boldsymbol{x}_{i}\right)-\hat{c}\left(\boldsymbol{x}_{i}\right)\right)^{2},
$$

where $\mathbb{I}(\boldsymbol{x})$ is the indicator function for whether the labeled input $\boldsymbol{x}$ is misclassified or not, i.e., $\mathbb{I}(\boldsymbol{x})=1$ if $\hat{y}(\boldsymbol{x})=y(\boldsymbol{x})$, and 0 otherwise.

Now we formally define the problem of operation calibration:
Problem. Given $\mathfrak{M}$ a previously trained DNN classifier, $S$ a set of $N$ unlabeled examples collected from an operation domain, and a budget $n \ll N$ for labeling the examples in $S$, the task of operational calibration is to find a confidence estimation function $\hat{c}(\cdot)$ for $\mathfrak{M}$ with minimal Brier score $B S(S)$.

Notice that operational calibration is different from the confidence calibration discussed in Section 3.1. The latter is domainindependent and usually included as a step in the training process of a DNN model (one of machine learning's focuses), but the former is needed when the model is deployed as a software component by a third party in a specific operation domain (what software engineering cares about). Technically, operational calibration cannot take the confidence error as a systematic error of the learning process, because the error is caused by the domain shift from the training data to the operation data, and it may assign different confidence values to inputs with the same uncalibrated confidence value.

\section{SOLVING OPERATIONAL CALIBRATION WITH GAUSSIAN PROCESS REGRESSION}

At first glance operational calibration seems a simple regression problem with BS as the loss function. However, a direct regression would not work because of the limited budget of labeled operation data. It is helpful to view the problem in a Bayesian way. At the beginning, we have a prior belief about the correctness of a DNN model's predictions, which is the confidence outputs of the model. Once we observe some evidences that the model makes correct or incorrect predictions on some inputs, the belief should be adjusted accordingly. The challenge here is to strike a balance between the priori that was learned from a huge training dataset but suffering from domain shift, and the evidence that is collected from the operation domain but limited in volume.

\subsection{Modeling with Gaussian Process}

It is natural to model the problem as a Gaussian Process [46], because what we need is actually a function $\hat{c}(\cdot)$. Gaussian Process is a non-parametric kind of Bayesian methods, which convert a prior over functions into a posterior over functions according to observed data.

For convenience, instead of estimating $\hat{c}(\cdot)$ directly, we consider

$$
h(\boldsymbol{x})=\hat{c}(\boldsymbol{x})-c_{\mathfrak{M}}(\boldsymbol{x}),
$$

where $c_{\mathfrak{M}}(\boldsymbol{x})$ is the original confidence output of $\mathfrak{M}$ for input $\boldsymbol{x}$. At the beginning, without any evidence against $c_{\mathfrak{M}}(\boldsymbol{x})$, we assume that the prior distribution of $h(\cdot)$ is a zero-mean normal distribution

$$
h \sim \mathcal{N}(\cdot \mid 0, k(\cdot, \cdot)),
$$

where $k(\cdot, \cdot)$ is the covariance (kernel) function, which intuitively describes the "smoothness" of $h(x)$ from point to point. In other words, the covariance function ensures that $h$ produces close outputs when inputs are close in the input space.

Assume that we observe a set of independent and identically distributed (i.i.d.) labeled operation data $I=\left\{\left(x_{i}, y_{i}\right) \mid 1 \leq i \leq n\right\}$, in which $y=h(\boldsymbol{x})=\mathbb{I}(\boldsymbol{x})-c_{\mathfrak{M}}(\boldsymbol{x})$. For notational convenience, let

$$
\begin{aligned}
\boldsymbol{X} & =\left(\boldsymbol{x}_{1}^{\top} ; \ldots ; \boldsymbol{x}_{n}^{\mathrm{\top}}\right) \quad \text { and } \\
\boldsymbol{h} & =\left(h\left(\boldsymbol{x}_{1}\right) ; \ldots ; h\left(\boldsymbol{x}_{n}\right)\right)
\end{aligned}
$$


be the observed data and their corresponding $y$-values, and let

$$
\begin{aligned}
\boldsymbol{X}^{\prime} & =\left(\left(\boldsymbol{x}_{1}^{\prime}\right)^{\mathrm{T}} ; \ldots ;\left(\boldsymbol{x}_{n}^{\prime}\right)^{\mathrm{T}}\right), \quad \text { and } \\
\boldsymbol{h}^{\prime} & =\left(h\left(\boldsymbol{x}_{1}^{\prime}\right) ; \ldots ; h\left(\boldsymbol{x}_{n}^{\prime}\right)\right)
\end{aligned}
$$

be those for a set $T=\left\{\left(\boldsymbol{x}_{i}^{\prime}, y_{i}^{\prime}\right), i=1, \ldots, m\right\}$ of i.i.d. predictive points. We have

$$
\left(\begin{array}{l}
\boldsymbol{h} \\
\boldsymbol{h}^{\prime}
\end{array}\right) \mid \boldsymbol{X}, \boldsymbol{X}^{\prime} \sim \mathcal{N}\left(\mathbf{0},\left(\begin{array}{cc}
K_{X X} & K_{X X^{\prime}} \\
K_{X^{\prime} X} & K_{X^{\prime} X^{\prime}}
\end{array}\right)\right)
$$

where $K$ is the kernel matrix. Therefore, the conditional probability distribution is

$$
\boldsymbol{y}^{\prime} \mid \boldsymbol{y}, X, X^{\prime} \sim \mathcal{N}\left(\boldsymbol{\mu}^{\prime}, \Sigma^{\prime}\right)
$$

where

$$
\begin{aligned}
\mu^{\prime} & =K_{X^{\prime} X}\left(K_{X X}\right)^{-1} y \\
\Sigma^{\prime} & =K_{X^{\prime} X^{\prime}}-K_{X^{\prime} X}\left(K_{X X}\right)^{-1} K_{X X^{\prime}}
\end{aligned}
$$

With this Gaussian Process, we can estimate the probability distribution of the operational confidence for any input $\boldsymbol{x}^{\prime}$ as follows

$$
h\left(\boldsymbol{x}^{\prime}\right) \mid \boldsymbol{x}^{\prime}, \boldsymbol{X}, \boldsymbol{h} \sim \mathcal{N}(\mu, \sigma),
$$

where

$$
\begin{aligned}
& \mu=K_{\boldsymbol{x}^{\prime} \boldsymbol{X}}\left(K_{X X}\right)^{-1} \boldsymbol{h}, \\
& \sigma=K_{\boldsymbol{x}^{\prime} \boldsymbol{x}^{\prime}}-K_{\boldsymbol{x}^{\prime} \boldsymbol{X}}\left(K_{X X}\right)^{-1} K_{X \boldsymbol{x}^{\prime}} .
\end{aligned}
$$

Then, with Equation 6, we have the distribution of $\hat{c}\left(\boldsymbol{x}^{\prime}\right)$

$$
P\left(\hat{c}\left(x^{\prime}\right) \mid x^{\prime}\right) \sim \mathcal{N}\left(c_{\mathfrak{M}}\left(x^{\prime}\right)+\mu, \sigma\right) .
$$

Finally, due to the value of confidence ranges from 0 to 1 , we need to truncate the original normal distribution [5], i.e.,

$$
P\left(\hat{c}\left(x^{\prime}\right) \mid x^{\prime}\right) \sim \mathcal{T} \mathcal{N}\left(\mu_{t n}, \sigma_{t n} ; \alpha, \beta\right),
$$

where

$$
\begin{aligned}
& \mu_{t n}=c_{\mathfrak{M}}\left(x^{\prime}\right)+\mu+\frac{\phi(\alpha)-\phi(\beta)}{\Phi(\alpha)-\Phi(\beta)} \sigma, \\
& \sigma_{t n}^{2}=\sigma^{2}\left[1+\frac{\alpha \phi(\alpha)-\beta \phi(\beta)}{\Phi(\beta)-\Phi(\alpha)}-\left(\frac{\phi(\alpha)-\phi(\beta)}{\Phi(\beta)-\Phi(\alpha)}\right)^{2}\right], \\
& \alpha=\left(0-c_{\mathfrak{M}}\left(\boldsymbol{x}^{\prime}\right)-\mu\right) / \sigma, \quad \beta=\left(1-c_{\mathfrak{M}}\left(\boldsymbol{x}^{\prime}\right)-\mu\right) / \sigma .
\end{aligned}
$$

Here the $\phi(\cdot)$ and $\Phi(\cdot)$ are the probability density function and the cumulative distribution function of standard normal distribution, respectively.

With this Bayesian approach, we compute a distribution, rather than an exact value, for the confidence of each prediction. To compute the Brier score, we simply choose the maximum a posteriori (MAP), i.e., the mode of the distribution, as the calibrated confidence value. Here it is the mean of the truncated normal distribution

$$
\hat{c}(\boldsymbol{x})=\mu_{t n} \text {. }
$$

\subsection{Clustering in Representation Space}

Directly applying the above Gaussian Process to estimate $\hat{c}(\cdot)$ would be ineffective and inefficient. It is difficult to specify a proper covariance function in Equation 7, because the correlation between the correctness of predictions on different examples in the very high-dimensional input space is difficult, if possible, to model.

Fortunately, we have the DNN model $\mathfrak{M}$ on hand, which can be used as a feature extractor, although it may suffer from the problem of domain shift [1]. In this way we transform each input $\boldsymbol{x}$ from the input space to a corresponding point $z$ in the representation space, which is defined by the output of the neurons in the last hidden layer. It turns out that the correctness of $\mathfrak{M}$ 's predictions has an obvious locality, i.e., a prediction is more likely to be correct/incorrect if it is near to a correct/incorrect prediction in the representation space.

Another insight for improving the efficacy and efficiency of the Gaussian Process is that the distribution of operation data in the sparse representation space is far from even. They can be nicely grouped into a small number (usually tens) of clusters, and the correlation of prediction correctness within a group is much stronger than that between groups. Consequently, instead of regression with a universal Gaussian Process, we carry out a Gaussian Process regression in each cluster.

This clustering does not only reduce the computational cost of the Gaussian Processes, but also make it possible to use different covariance functions for different clusters. The flexibility makes our estimation more accurate. Elaborately, we use the RBF kernel

$$
k\left(z_{1}, z_{2}\right)=\exp \left(-\frac{\left\|z_{1}-z_{2}\right\|^{2}}{2 \ell^{2}}\right)
$$

where the parameter $\ell$ (length scale) can be decided according to the distribution of the original confidence produced by $\mathfrak{M}$.

\subsection{Considering Costs in Decision}

The cost of misclassification must be taken into account in realworld decision making. We propose to also measure how well a model is calibrated with the loss due to confidence error (LCE) against a given cost model.

For example, let us assume a simple cost model in which the gain for a correct prediction is 1 and the loss for a false prediction is $u$. The net gain if we take action on a prediction for input $x$ will be $\mathbb{I}(\boldsymbol{x})-u \cdot(1-\mathbb{I}(\boldsymbol{x}))$. We further assume that there will be no cost to take no action when the expected net gain is negative. Then the actual gain for an input $\boldsymbol{x}$ with estimated confidence $\hat{c}(\boldsymbol{x})$ will be

$$
g(\boldsymbol{x})=\left\{\begin{array}{cl}
\mathbb{I}(\boldsymbol{x})-u \cdot(1-\mathbb{I}(\boldsymbol{x})) & \text { if } \hat{c}(\boldsymbol{x}) \geq \lambda, \\
0 & \text { if } \hat{c}(\boldsymbol{x})<\lambda,
\end{array}\right.
$$

where $\lambda=\frac{u}{1+u}$ is the break-even threshold of confidence for taking action. On the other hand, if the confidence was perfect, i.e., $\hat{c}(\boldsymbol{x})=1$ if the prediction was correct, and 0 otherwise, the total gain for dataset $D$ would be a constant $G_{D}=\sum_{i=1}^{N} \mathbb{I}\left(\boldsymbol{x}_{i}\right)$. So the average LCE over a dataset $D$ with $N$ examples is $\ell(D)=\frac{1}{N}\left(G_{D}-\sum_{i=1}^{N} g\left(\boldsymbol{x}_{i}\right)\right)$.

With the Bayesian approach we do not have an exact $\hat{c}(\boldsymbol{x})$ but a truncated normal distribution of it. If we take $\mu_{t n}(\boldsymbol{x})$ as $\hat{c}(\boldsymbol{x})$, the above equations still hold.

Cost-sensitive calibration targets at minimizing the LCE instead of the Brier score. Notice that calibrating confidence with Brier score generally reduces LCE. However, with a cost model, the optimization toward minimizing LCE can be more effective and efficient.

\subsection{Selecting Operation Data to Label}

In case that the set of labeled operation data is given, we simply apply a Gaussian Process in each cluster in the representation space and get the posteriori distribution for confidence $\hat{c}(\cdot)$. However, if we can decide which operation data to label, we shall spend the budget for labeling more wisely. 
Initially, we select the operational input at the center of each cluster to label, and apply a Gaussian Process in each cluster with this central input to compute the posterior probability distribution of the confidence. Then we shall select the most "helpful" input to label and repeat the procedure. The insight for input selection is twofold. First, to reduce the uncertainty as much as possible, one should choose the input with maximal variance $\sigma_{t n}^{2}$. Second, to reduce the LCE as much as possible, one should pay more attention to those input with confidence near to the break-even threshold $\lambda$. So we chose $\boldsymbol{x}^{*}$ as the next input to label:

$$
\boldsymbol{x}^{*}=\arg \min _{\boldsymbol{x}} \frac{\left|\mu_{t n}(\boldsymbol{x})-\lambda\right|}{\sigma_{t n}(\boldsymbol{x})} .
$$

With $x^{*}$ and its label $y^{*}$, we update the corresponding Gaussian Process model and get better $\mu_{t n}(\cdot)$ and $\sigma_{t n}(\cdot)$. The select-labelupdate procedure is repeated until the labeling budget is used up.

Putting all the ideas together, we have Algorithm 1 shown below. The algorithm is robust in that it does not rely on any hyperparameters except for the number of clusters. It is also conservative in that it does not change the predictions made by the model. As a result, it needs no extra validation data.

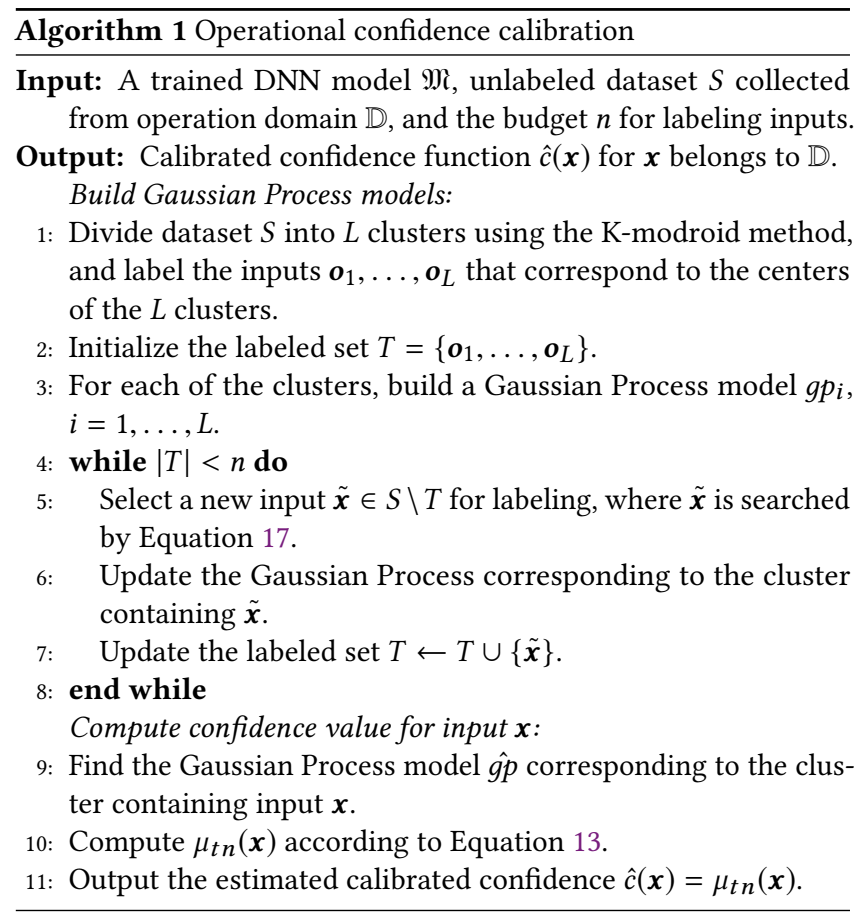

\subsection{Discussions}

To understand why our approach is more effective than conventional confidence calibration techniques, one can consider the threepart decomposition of the Brier score [32]

$$
\begin{aligned}
B S= & \sum_{m=1}^{M} \frac{\left|D_{m}\right|}{N}\left(\operatorname{conf}\left(D_{m}\right)-\operatorname{acc}\left(D_{m}\right)\right)^{2} \\
& -\sum_{m=1}^{M} \frac{1}{N}\left(\operatorname{acc}\left(D_{m}\right)-\operatorname{acc}\right)^{2}+\operatorname{acc}(1-\operatorname{acc}),
\end{aligned}
$$

where $D_{m}$ is the set of inputs whose confidence falls into the interval $I_{m}=\left(\frac{m-1}{M}, \frac{m}{M}\right]$, and the $\operatorname{acc}\left(D_{m}\right)$ and $\operatorname{conf}\left(D_{m}\right)$ are the expected accuracy and confidence in $D_{m}$, respectively. The acc is the accuracy of dataset $D$.

In this decomposition, the first term is called reliability, which measures the distance between the confidence and the true posterior probabilities. The second term is resolution, which measures the distinctions of the predictive probabilities. The final term is uncertainty, which is only determined by the accuracy.

In conventional confidence calibration, the model is assumed to be well trained and work well with the accuracies. In addition, the grouping of $D_{m}$ is acceptable because the confidence error is regarded as systematic error. So one only cares about minimizing the reliability. This is exactly what conventional calibration techniques such as Temperature Scaling are designed for.

However, in operational calibration, the model itself suffers from the domain shift, and thus may be less accurate than expected. Even worse, the grouping of $D_{m}$ is problematic because the confidence error is unsystematic and the inputs in $D_{m}$ are not homogeneous anymore. Consequently, we need to maximize the resolution and minimize the reliability at the same time. Our approach achieves these two goals with more discriminative calibration that is based on the features of individual inputs rather than their logits or confidence values.

This observation also indicates that the benefit of our approach over Temperature Scaling will diminish if the confidence error happens to be systematic. For example, in case that the only divergence of the data in the operation domain is that some part of an image is missing, our approach will perform similarly to or even slightly worse than Temperature Scaling. However, as can be seen from later experiments, most operational situations have more or less domain shifts that Temperature Ccaling cannot handle well.

In addition, when the loss for false prediction $u$ is very small ( $u \leq 0.11$, as observed from experiments in the next section), our approach will be ineffective in reducing LCE. It is expected because in this situation one should accept almost all predictions, even when their confidence values are low.

\section{EMPIRICAL EVALUATION}

We conducted a series of experiments to answer the following questions:

(1) Is our approach to operational calibration generally effective in different tasks?

(2) How effective it is, compared with alternative approaches?

(3) How efficient it is, in the sense of saving labeling efforts?

We implemented our approach on top of the PyTorch 1.1.0 DL framework. The code, together with the experimental data, are available at https://github.com/Lizn-zn/Op-QA. The experiments were conducted on a GPU server with two Intel Xeon Gold 5118 CPU @ 2.30GHz, 400GB RAM, and 10 GeForce RTX 2080 Ti GPUs. The server ran Ubuntu 16.04 with GNU/Linux kernel 4.4.0.

The execution time of our operational calibration depends on the size of the dataset used, and the architecture of the DNN model. For the tasks listed below, the execution time varied from about $3.5 \mathrm{~s}$ to $50 \mathrm{~s}$, which we regard as totally acceptable. 


\subsection{Experimental Tasks}

To evaluate the general efficacy of our approach, we designed six tasks that were different in the application domains (image recognition and natural language processing), operation dataset size (from hundreds to thousands), classification difficulty (from 2 to 1000 classes), and model complexity (from $\sim 10^{3}$ to $\sim 10^{7}$ parameters). To make our simulation of domain shifts realistic, in four tasks we adopted third-party operation datasets often used in the transfer learning research, and the other two tasks we used mutations that are also frequented made in the machine learning community. Figure 2 demonstrates some example images from the origin and operation domains for tasks 3 and 5 . Table 1 lists the settings of the six tasks.

Table 1: Dataset and model settings of tasks

\begin{tabular}{|c|c|c|c|c|}
\hline \multirow{2}{*}{ Task } & \multirow{2}{*}{ Model } & \multicolumn{3}{|c|}{ Origin Domain $\rightarrow$ Operation Domain } \\
\cline { 3 - 5 } & LeNet-5 & $\begin{array}{c}\text { Digit recognition } \\
\text { (MNIST } \rightarrow \text { USPS) }\end{array}$ & $96.9 \rightarrow 68.0$ & 900 \\
\hline \hline 1 & RNN & $\begin{array}{c}\text { Polarity } \\
\text { (v1.0 } \rightarrow \text { v2.0) }\end{array}$ & $99.0 \rightarrow 83.4$ & 1,000 \\
\hline 3 & ResNet-18 & $\begin{array}{c}\text { Image classification } \\
\text { CIFAR-10 } \rightarrow \text { STL-10 }\end{array}$ & $93.2 \rightarrow 47.1$ & 5,000 \\
\hline 4 & VGG-19 & $\begin{array}{c}\text { CIFAR-100 } \\
\text { (orig. } \rightarrow \text { crop) }\end{array}$ & $72.0 \rightarrow 63.6$ & 5,000 \\
\hline 5 & ResNet-50 & $\begin{array}{c}\text { ImageCLEF } \\
\text { (c } \rightarrow \text { p) }\end{array}$ & $99.2 \rightarrow 73.2$ & 480 \\
\hline 6 & Inception-v3 & $\begin{array}{c}\text { ImageNet } \\
\text { (orig. } \rightarrow \text { down-sample) }\end{array}$ & $77.5 \rightarrow 45.3$ & 5,000 \\
\hline
\end{tabular}

It refers to the maximum number of operation data available for labeling.

In Task 1 we applied a LeNet-5 model originally trained with the images from the MNIST dataset [24] to classify images from the USPS dataset [11]. Both of them are popular handwritten digit recognition datasets consisting of single-channel images of size $16 \times 16 \times 1$, but the latter is more difficult to read. The size of the training dataset was 2,000 , and the size of the operation dataset was 1,800 . We reserved 900 of the 1,800 operation data for testing, and used the other 900 for operational calibration.

Task 2 was focused on natural language processing. Polarity is a dataset for sentiment-analysis [39]. It consists of sentences labeled with corresponding sentiment polarity (i.e., positive or negative). We chose Polarity-v1.0, which contained 1,400 movie reviews collected in 2002, as the training set. The Polarity-v2.0, which contained 2,000 movie reviews collected in 2004, was used as the data from the operation domain. We also reserved half of the operation data for testing.

In Task 3 we used two classic image classification datasets CIFAR10 [21] and STL-10 [7]. The former consists of $60,00032 \times 32 \times 3 \mathrm{im}-$ ages in 10 classes, and each class contains 6,000 images. The latter has only 1,3000 images, but the size of each image is $96 \times 96 \times 3$. We used the whole CIFAR-10 dataset to train the model. The operation domain was represented by 8,000 images collected from STL-10, in which 5,000 were used for calibration, and the other 3,000 were reserved for testing.
Tasks 4 used the dataset CIFAR-100, which was more difficult than CIFAR-10 and contained 100 classes with 600 images in each. We trained the model with the whole training dataset of 50,000 images. To construct the operation domain, we randomly cropped the remaining 10,000 images. One half of these cropped images were used for calibration and the other half for testing.

Task 5 used the image classification dataset from the ImageCLEF 2014 challenge [30]. It is organized with 12 common classes derived from three different domains: ImageNet ILSVRC 2012 (i), Caltech-256 (c), and Pascal VOC 2012 (p). We chose the dataset (c) as the origin domain and dataset (p) as the operation domain. Due to the extremely small size of the dataset, we divided the dataset (p) for calibration and testing by the ratio 4:1.

Finally, Task 6 dealt with an extremely difficult situation. ImageNet is a large-scale image classification dataset containing more than 1.2 million $224 \times 224 \times 3$ images across 1,000 categories [9]. The pre-trained model Inception-v3 was adopted for evaluation. The operation domain was constructed by down-sampling 10,000 images from the original test dataset. Again, half of the images were reserved for testing.

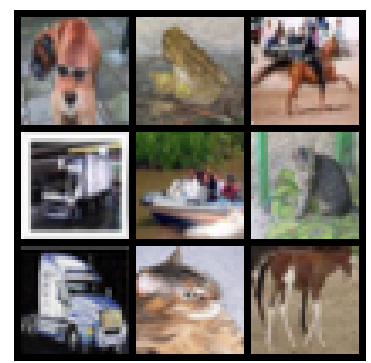

(a) CIFAR-10 (origin domain)

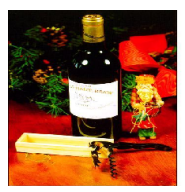

(c) ImageCLEF-(c) (origin domain)

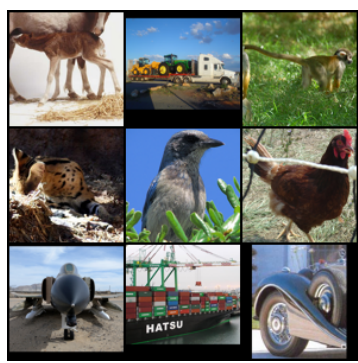

(b) STL-10 (operation domain)

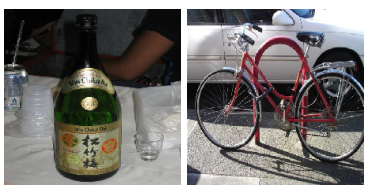

(d) ImageCLEF-(p) (operation domain)
Figure 2: Examples of origin and operation domains. In task 3 a ResNet-18 model was trained with low-resolution images (a), but applied to high-resolution images (b). In task 5 a ResNet-50 model was applied to images (d) with backgrounds and styles different from training images (c).

\subsection{Efficacy of Operational Calibration}

Table 2 gives the Brier scores of the confidence before (col. Orig.) and after (col. GPR) operational calibration. In these experiments all operation data listed in Table 1 (not including the reserved test data) were labeled and used in the calibration. The result unambiguously confirmed the general efficacy of our approach. In the following we elaborate on its relationship with the fine-tuning technique often employed in practice. 
Table 2: Brier scores of different calibration methods

\begin{tabular}{|c|c|c|c|c|c|c|c|c|c|c|}
\hline \multirow{2}{*}{ Task } & \multirow{2}{*}{ Model } & \multirow{2}{*}{ Orig. } & \multicolumn{3}{|c|}{ Operational calibration } & \multicolumn{4}{|c|}{ Conventional calibration } & \multirow{2}{*}{ SAR } \\
\cline { 5 - 10 } & & & GPR & RFR & SVR & TS & PS-conf. & PS-logit & IR & \\
\hline \hline 1 & LeNet-5 & 0.207 & $\mathbf{0 . 1 1 4}$ & 0.126 & 0.163 & 0.183 & 0.316 & 0.182 & 0.320 & 0.320 \\
\hline 2 & RNN & 0.203 & $\mathbf{0 . 1 0 2}$ & 0.107 & 0.202 & 0.185 & 0.641 & 0.125 & 0.655 & 0.175 \\
\hline 3 & ResNet-18 & 0.474 & $\mathbf{0 . 1 0 1}$ & 0.121 & 0.115 & 0.387 & 0.471 & 0.254 & 0.529 & 0.308 \\
\hline 4 & VGG-19 & 0.216 & $\mathbf{0 . 1 5 8}$ & 0.162 & 0.170 & 0.217 & 0.359 & 0.253 & 0.364 & 0.529 \\
\hline 5 & ResNet-50 & 0.226 & $\mathbf{0 . 1 7 9}$ & 0.204 & 0.245 & 0.556 & 0.789 & 0.319 & 0.925 & 0.364 \\
\hline 6 & Inception-v3 & 0.192 & $\mathbf{0 . 1 6 1}$ & 0.167 & 0.217 & 0.191 & 0.546 & 0.427 & 0.334 & - \\
\hline
\end{tabular}

Orig.-Before calibration. GPR-Gaussian Process-based approach. RFR-Random Forest Regression in the representation space. SVRSupport Vector Regression in the representation space. TS-Temperature Scaling [14]. PS-Platt Scaling [45]. IR-Isotonic Regression Scaling [65]. Conf./Logit indicates that the calibration took confidence value/logit as input. SAR-Regression with Surprise values [19]. We failed to evaluate SAR on task 6 because it took too long to run on the huge dataset.

5.2.1 Calibration when fine-tuning is ineffective. A machine learning engineer might first consider to apply fine-tuning tricks to deal with the problem of domain shift. However, for non-trivial tasks, such as our tasks 4,5 , and 6 , it can be very difficult, if possible, to fine-tune the DNN model with small operation datasets. Figure 3 shows the vain effort in fine-tuning the models with all the operation data (excluding test data). We tried all tricks including data augmentation, weight decay, and regularization to avoid over-fitting but failed to improve the test accuracy.

Fortunately, our operational calibration worked quite well in these difficult situations. In addition to the improvement in Brier scores reported in Table 2, we can also see the saving of LCE for task 4 in Figure 4 as an example. Our approach reduced about a half of the LCE when $\lambda>0.8$, which indicates its capability in reducing high confidence errors.

5.2.2 Calibration when fine-tuning is effective. In case of easier situations that fine-tuning works, we can still calibrate the model to give more accurate confidence. Note that effective fine-tuning does not necessarily provide accurate confidence. One can first apply fine-tuning until test accuracy does not increase, and then calibrate the fine-tuned model with the rest operation data.

For example, we managed to fine-tune the models in our tasks 1, 2, and 3. ${ }^{1}$ Task 1 was the easiest to fine-tune and its accuracy kept increasing and exhausted all the 900 operational examples. Task 2 was binary classification, in this case our calibration was actual an effective fine-tuning technique. Figure 5 a shows that our approach was more effective and efficient than conventional finetuning as it converged more quickly. For task 3 with fine-tuning the accuracy stopped increasing at about $79 \%$, with about 3,000 operational examples. Figure $5 \mathrm{~b}$ show that, the Brier score would decrease more if we spent rest operation data on calibration than continuing on the fine-tuning.

Based on the significant (16.1\%-78.6\%) reductions in Brier scores in all of the tasks reported in Table 2 and the above discussions, we conclude that the Gaussian Process-based approach to operational calibration is generally effective, and it is worthwhile no matter whether the fine-tuning works or not.

\footnotetext{
${ }^{1}$ Here we used some information of the training process, such as the learning rates, weight decays and training epochs. Fine-tuning could be more difficult because these information could be unavailable in real-world operation settings.
}

\subsection{Comparing with Alternative Methods}

First, we applied three widely used calibration methods, viz. Temperature Scaling (TS) [16], Platt Scaling (PS) [45], and Isotonic Regression (IR) [65], with the same operation data used in our approach. TS defines the calibration function $R$ in Equation 4 as $R(\boldsymbol{h})=\boldsymbol{h} / T$, where $T$ is a scalar parameter computed by minimizing the negative log likelihood [15] on a validation dataset. PS and IR directly work on the confidence values. PS trains a one-dimensional logistic regression and calibrates confidence as $\hat{c}=1 /\left(1+e^{-a \cdot c_{M}+b}\right)$, where $a, b$ are scalar parameters computed by minimizing the cross entropy on a validation dataset. IR simply fits a monotonic confidence calibration function minimizing the Brier score on a validation dataset. Note that in our experiment, the operation data were used instead of the validated dataset. We implemented TS according to Guo et al. [14]. For PS and IR we used the well-known machine learning library scikit-learn [42].

As shown in Table 2, TS, although reported to be usually the most effective conventional confidence calibration method [14], was hardly effective in these cases. It even worsened the confidence in tasks 4 and 5 . We observed that its bad performance came from the significantly lowered resolution part of the Brier score, which confirmed the analysis in Section 4.5. For example, in task 3, with Temperature Scaling the reliability decreased from 0.196 to 0.138 , but the resolution dropped from 0.014 to 0.0 . In fact, in this case the calibrated confidence values were all very closed to 0.5 after scaling. However, with our approach the reliability decreased to 0.107 , and the resolution also increased to 0.154 . The same reason also failed PS (col. PS-Conf.) and IR (col. IR).

We also included in our comparison an improved version of PS, which built a regression over the logit instead of the confidence [14]. Its calibration function $R$ for Equation 4 was $R(\boldsymbol{h})=\boldsymbol{W}^{\top} \boldsymbol{h}+\boldsymbol{b}$, where $\boldsymbol{W}$ and $\boldsymbol{b}$ were computed by minimizing the cross entropy on the operation data. It performed much better than the original PS but still failed in tasks 4,5 and 6 .

Second, we also tried to calibrate confidence based on the Surprise value that measured the difference in DL system's behavior between the input and the training data [19]. We thought it could be effective because it also leveraged the distribution of examples in the representation space. We made polynomial regression between the confidence adjustments and the likelihood-based Surprise values. Unfortunately, it did not work for most of the cases (col. SAR 


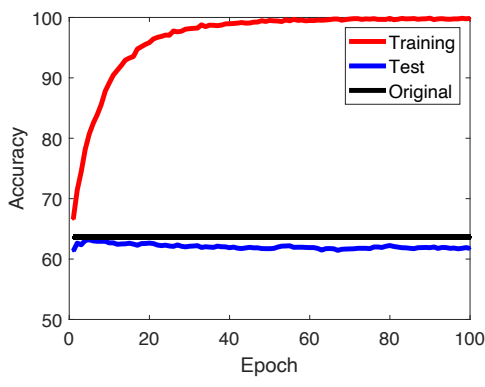

(a) Task 4

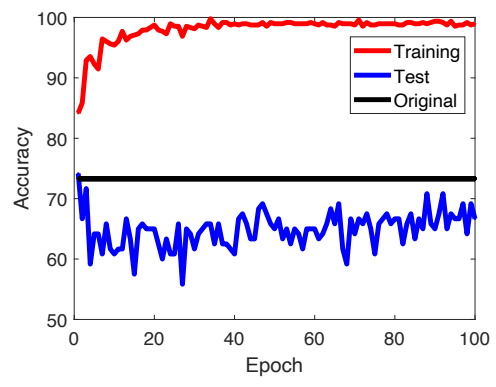

(b) Task 5

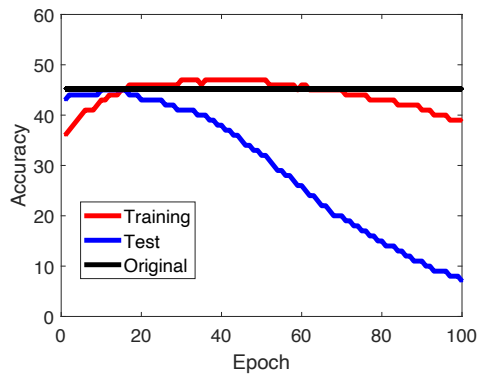

(c) Task 6

Figure 3: Ineffective fine-tuning of difficult tasks. All available operation data (5000, 480, and 5000, respectively) were used.

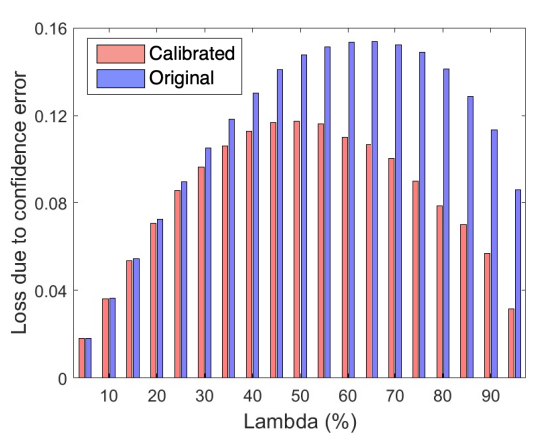

Figure 4: loss due to confidence error

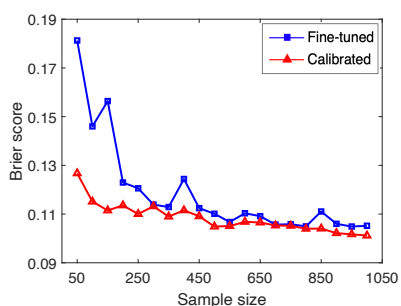

(a) Task 2

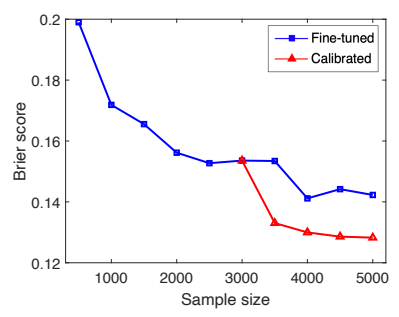

(b) Task 3
Figure 5: Calibration when fine-tuning is effective

in Table 2 ). We believe the reason is that Surprise values are scalars and cannot provide enough information for operational calibration.

Finally, to examine whether Gaussian Process Regression (GPR) is the right choice for our operational calibration framework, we also experimented with two standard regression methods, viz. Random Forest Regression (RFR) and Support Vector Regression (SVR). We used linear kernel for SVR and ten decision trees for RFR. As shown in Table 2, in most cases, the non-liner RFR performed better than the linear SVR, and both of them performed better than Temperature Scaling but worse than GPR. The result indicates that (1) calibration based on the features extracted by the model rather than the logits computed by the model is crucial, (2) the confidence error is non-linear and unsystematic, and (3) the Gaussian Process as a Bayesian method can provide better estimation of the confidence.

In summary, our GPR approach achieved significant Brier Score reduction and outperformed conventional calibration methods and the Surprise value-base regression in all the tasks. It also outperformed alternative implementations based on RFR and SVR. So we conclude that our approach is more effective than Temperature Scaling and other alternative choices for operational calibration.

\subsection{Efficiency of Operational Calibration}

In the above we have already shown that our approach worked with small operation datasets that were insufficient for fine-tuning (Task 4, 5, and 6). In fact, the Gaussian Process-based approach has a nice property that it starts to work with very few labeled examples. We experimented the approach with the input selection method presented in Section 4.4. We focused on the number of high-confidence false predictions, which was decreasing as more and more operational examples were labeled and used.

We experimented with all the tasks but labeled only $10 \%$ of the operation data. Table 3 shows the numbers of high-confidence false predictions before and after operational calibration. As a reference, we also include the numbers of high-confidence correct predictions. We can see that most of the high-confidence false predictions were eliminated. It is expected that there were less high-confidence correct predictions after calibration, because the actual accuracy of the models dropped. The much lowered LCE scores, which took into account both the loss in lowering the confidence of correct predictions and the gain in lowering the confidence of false predictions, indicate that the overall improvements were significant.

For a visual illustration of the efficiency of our approach, Figure 6 plots the change of proportions of high-confidence false and correct predictions as the size of data used in calibration increases. It is interesting to see that: (1) most of the high-confidence false predictions were identified very quickly, and (2) the approach was conservative, but the conservativeness is gradually remedied with more labeled operation data used.

Note that for tasks 4, 5 and 6, usual fine-tuning tricks did not work even with all the operation data labeled. With our operational 
Table 3: Reducing high-confidence false predictions with $10 \%$ operation data labeled

\begin{tabular}{|c|c|c|c|c|c|}
\hline No. & Model & $\lambda$ & Correct pred. & False pred. & LCE \\
\hline \hline \multirow{2}{*}{1} & \multirow{2}{*}{ LeNet-5 } & 0.8 & $473 \rightarrow 309.1$ & $\mathbf{1 2 6} \rightarrow \mathbf{2 4 . 3}$ & $0.143 \rightarrow 0.089$ \\
& 0.9 & $417 \rightarrow 141.9$ & $\mathbf{7 4} \rightarrow \mathbf{2 . 5}$ & $0.096 \rightarrow 0.055$ \\
\hline \multirow{2}{*}{2} & \multirow{2}{*}{ RNN } & 0.8 & $512 \rightarrow 552.9$ & $\mathbf{1 1 8} \rightarrow \mathbf{3 9 . 9}$ & $0.162 \rightarrow 0.091$ \\
& & 0.9 & $482 \rightarrow 261.3$ & $\mathbf{1 0 6} \rightarrow \mathbf{1 2 . 0}$ & $0.132 \rightarrow 0.070$ \\
\hline \multirow{2}{*}{3} & ResNet & 0.8 & $1350 \rightarrow 839.2$ & $\mathbf{1 3 7 2} \rightarrow \mathbf{5 9 . 7}$ & $0.370 \rightarrow 0.054$ \\
& -18 & 0.9 & $1314 \rightarrow 424.0$ & $\mathbf{1 2 6 3} \rightarrow \mathbf{9 . 4}$ & $0.358 \rightarrow 0.041$ \\
\hline \multirow{2}{*}{4} & \multirow{2}{*}{ VGG-19 } & 0.8 & $1105 \rightarrow 392.5$ & $\mathbf{5 8 3 \rightarrow \mathbf { 4 6 . 9 }}$ & $0.127 \rightarrow 0.070$ \\
& & 0.9 & $772 \rightarrow 142.8$ & $\mathbf{2 8 0} \rightarrow \mathbf{9 . 3}$ & $0.074 \rightarrow 0.038$ \\
\hline \multirow{2}{*}{5} & ResNet & 0.8 & $53 \rightarrow 26.9$ & $\mathbf{1 6} \rightarrow \mathbf{5 . 2}$ & $0.162 \rightarrow 0.136$ \\
& -50 & 0.9 & $46 \rightarrow 26.9$ & $\mathbf{1 0} \rightarrow \mathbf{2 . 0}$ & $0.108 \rightarrow 0.064$ \\
\hline \multirow{2}{*}{6} & Inception & 0.8 & $1160 \rightarrow 692.0$ & $\mathbf{2 6 5} \rightarrow \mathbf{6 3 . 6}$ & $0.087 \rightarrow 0.073$ \\
& $-v 3$ & 0.9 & $801 \rightarrow 554.1$ & $\mathbf{1 3 7} \rightarrow \mathbf{4 0 . 2}$ & $0.054 \rightarrow 0.041$ \\
\hline
\end{tabular}

We ran each experiment 10 times and computed the average numbers.

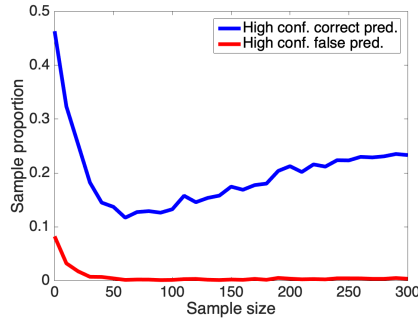

(a) Task 1

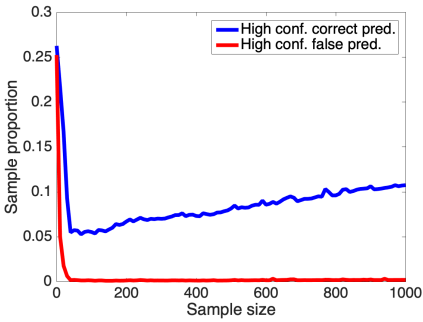

(c) Task 3

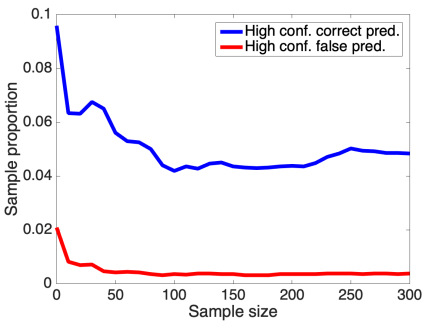

(e) Task 5

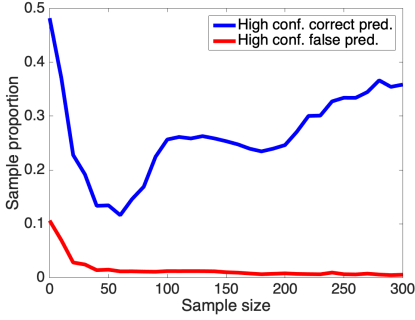

(b) Task 2

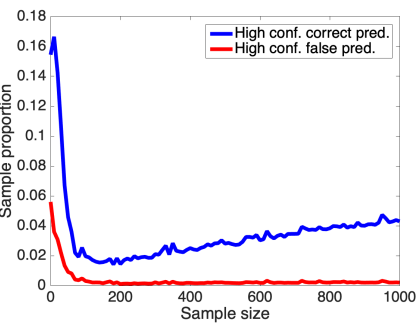

(d) Task 4

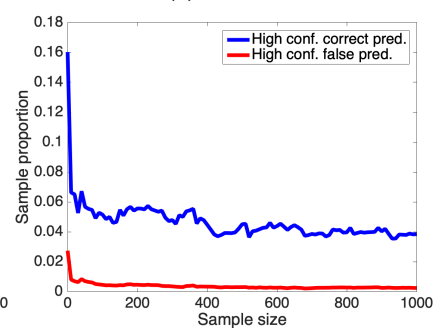

(f) Task 6
Figure 6: The proportion curve of high confidence inputs. Sample size 0 means uncalibrated. The calibration started to take effect with very few data.

calibration, using only about $10 \%$ of the data, we avoided about $97 \%, 80 \%$, and $71 \%$ high-confidence $(>0.9)$ errors, respectively.
Based on the results, we can say that the Gaussian Process-based operational calibration is efficient in detecting most of the highconfidence errors with a small amount of labeled operation data.

\section{RELATED WORK}

Operational calibration is generally related to the quality assurance for deep learning systems in the software engineering community, and the confidence calibration, transfer learning, and active learning in the machine learning community. We briefly overview related work in these directions and highlight the connections and differences between our work and them.

\subsection{Software Quality Assurance for Deep Learning Systems}

The research in this area can be roughly classified into four categories according to the kind of defects targeted:

- Defects in DL programs. This line of work focuses on the bugs in the code of DL frameworks. For example, Pham et al. proposed to test the implementation of deep learning libraries (TensorFlow, CNTK and Theano) through differential testing [44]. Odena et al. used fuzzing techniques to expose numerical errors in matrix multiplication operations [37].

- Defects in DL models. Regarding trained DNN models as pieces of software artifact, and borrowing the idea of structural coverage in conventional software testing, a series of coverage criteria have been proposed for the testing of DNNs, for example, DeepXplore [43], DeepGauge [29], DeepConcolic [56], and Surprise Adequacy [19], to name but a few.

- Defects in training datasets. Another critical element in machine learning is the dataset. There exist research aiming at debugging and fixing errors in the polluted training dataset. For example, PSI identifies root causes (e.g., incorrect labels) of data errors by efficiently computing the Probability of Sufficiency scores through probabilistic programming [6].

- Defects due to improper inputs. A DNN model cannot well handle inputs out of the distribution for which it is trained. Thus a defensive approach is to detect such inputs. For example, Wang et al.'s approach checked whether an input is normal or adversarial by integrating statistical hypothesis testing and model mutation testing [62]. Wang et al. proposed DISSECTOR, which effectively distinguished unexpected inputs from normal inputs by verifying progressive relationship between layers [60]. More work in this line can be found in the machine learning literature under the name of out-of-distribution detection [53].

For a more comprehensive survey on the testing of machine learning systems, one can consult Zhang et al. [67].

The main difference of our work, compared with these pieces of research, is that it is operational, i.e., focusing on how well a DNN model will work in a given operation domain. As discussed in Section 2, without considering the operation domain, it is often difficult to tell whether a phenomena of a DNN model is a bug or a feature $[17,25]$.

An exception is the recent proposal of operational testing for the efficient estimation of the accuracy of a DNN model in the field [26]. Arguably operational calibration is more challenging and more rewarding than operational testing, because the latter only 
tells the overall performance of a model in an operation domain, but the former tells when it works well and when not.

\subsection{DNN Confidence Calibration}

Confidence calibration is important for training high quality classifiers. There is a plethora of proposals on this topic in the machine learning literature [10, 14, 33, 35, 65]. Apart from the Temperature Scaling discussed in Section 3.1, Isotonic regression [65], Histogram binning [64], and Platt scaling [45] are also often used. Isotonic regression is a non-parametric approach that employs the least square method with a non-decreasing and piecewise constant fitted function. Histogram binning divides confidences into mutually exclusive bins and assigns the calibrated confidences by minimizing the bin-wise squared loss. Platt scaling is a generalized version of Temperature Scaling. It adds a linear transformation between the logit layer and the softmax layer, and optimizes the parameters with the NLL loss. However, according to Guo et al., Temperature Scaling is often the most effective approach [14].

As discussed earlier in Section 4.5, the problem of these calibration methods is that they regard confidence errors as systematic errors, which is usually not the case in the operation domain. Technically, these calibration methods are effective in minimize the reliability part of the Brier score, but ineffective in dealing with the problem in the resolution part.

In addition, Flach discussed the problem of confidence calibration from a decision-theoretic perspective [10]. However, the confidence error caused by domain shift was not explicitly addressed.

Previous research efforts on confidence calibration at prediction time instead of training time are uncommon, but do exist. Gal and Ghahramani proposed to build a temporary ensemble model by using dropout at prediction time [12]. Despite its elegant Bayesian inference framework, the method is computationally too expensive to handle large-scale tasks. Recently, based on the insight of conformal prediction [52], Papernot and McDaniel proposed to build a k-Nearest Neighbors $(\mathrm{kNN})$ model for the output of each DNN immediate layer [40]. The confidence of a prediction was estimated by the conformity of these kNNs' outputs. Unfortunately, this method is not applicable to non-trivial tasks either because of its incapability in handling high-dimensional examples. Note that these methods did not explicitly consider confidence errors caused by domain shifts.

Another related line of work is under the name of uncertainty estimation [22, 48, 49]. For example, Evidential Deep Learning qualitatively evaluates the uncertainty of DNN predictions with a Dirichlet distribution placed on the class probabilities [49]. However, these methods mainly aim at out-of-distribution detection, which is considered easier than confidence calibration [10]. Identifying out-ofdistribution inputs is useful in defending against adversarial attacks, but not directly helpful in adapting a model to a new operation domain.

\subsection{Transfer Learning and Active Learning}

Our approach to operational calibration borrowed ideas from transfer learning [38] and active learning [51]. Transfer learning (or domain adaptation) aims at training a model from a source domain (origin domain in our terms) that can be generalized to a target domain (operation domain), despite the dataset shift [34] between the domains. The key is to learn features that are transferable between the domains.

However, transfer learning techniques usually require data from both of the source and target domains. Contrastingly, operational calibration often has to work with limited data from the operation domain and no data from the origin domain. Transfer learning usually fails to work under this constraint. Even in case it works, it does not necessarily produce well calibrated models, and operational calibration is needed to correct confidence errors (cf. Figure 5b).

Active learning aims at reducing the cost of labeling training data by deliberately selecting and labeling inputs from a large set of unlabeled data. For the Gaussian Process Regression, there exist different input selection strategies $[18,41,50]$. We tried many of them, such as those based on uncertainty [50], on density [71], and on disagreement [41], but failed to find a reliable strategy that can further improve the data efficiency of our approach. They were very sensitive to the choices of the initial inputs, the models, and the distribution of examples [51]. However, we found that the combination of cost-sensitive sampling bias and uncertainty can help in reducing high-confidence false predictions, especially in a cost-sensitive setting.

\section{CONCLUSION}

Software quality assurance for systems incorporating DNN models is urgently needed. This paper focuses on the problem of operational calibration that detects and fixes the errors in the confidence given by a DNN model for its predictions in a given operation domain. A Bayesian approach to operational calibration is given. It solves the problem with Gaussian Process Regression, which leverages the locality of the operation data, and also of their prediction correctness, in the representation space. Experiments with representative datasets and DNN models confirmed that the approach can significantly reduce the risk of high-confidence false prediction with a small number of labeled data, and thus efficiently improve the models' quality of service in operational settings.

While with empirical evidence, we consider conducting more theoretical analysis on aspects such as the data efficiency and the convergence of our algorithm as future work. In addition, we plan to investigate operational calibration methods for real-world decisions with more complicated cost models.

\section{ACKNOWLEDGMENTS}

We thank the anonymous reviewers for their suggestions. This work is supported by the National Natural Science Foundation of China (61690204, 61932021, 61802170) and the Collaborative Innovation Center of Novel Software Technology and Industrialization.

\section{REFERENCES}

[1] Yoshua Bengio, Aaron C Courville, and Pascal Vincent. 2012. Unsupervised feature learning and deep learning: A review and new perspectives. CoRR, abs/1206.5538 1 (2012), 2012.

[2] Christopher M Bishop. 2006. Pattern recognition and machine learning. Springer, New York, NY. http://cds.cern.ch/record/998831 Softcover published in 2016.

[3] Mariusz Bojarski, Davide Del Testa, Daniel Dworakowski, Bernhard Firner, Beat Flepp, Prasoon Goyal, Lawrence D. Jackel, Mathew Monfort, Urs Muller, Jiakai Zhang, Xin Zhang, Jake Zhao, and Karol Zieba. 2016. End to End Learning 
for Self-Driving Cars. CoRR abs/1604.07316 (2016), 9. arXiv:1604.07316 http: //arxiv.org/abs/1604.07316

[4] Glenn W Brier. 1950. Verification of forecasts expressed in terms of probability. Monthly weather review 78, 1 (1950), 1-3.

[5] John Burkardt. 2014. The truncated normal distribution. , 32 pages. http //people.sc.fsu.edu/ jburkardt/presentations/truncatednormal.pdf

[6] Aleksandar Chakarov, Aditya Nori, Sriram Rajamani, Shayak Sen, and Deepak Vijaykeerthy. 2016. Debugging machine learning tasks. arXiv preprint arXiv:1603.07292 (2016), 23.

[7] Adam Coates, Andrew Ng, and Honglak Lee. 2011. An analysis of single-layer networks in unsupervised feature learning. In Proceedings of the fourteenth international conference on artificial intelligence and statistics. aistats, 215-223.

[8] Council of European Union. 2014. Council regulation (EU) no 269/2014. http://eur-lex.europa.eu/legal-content/EN/TXT/?qid=1416170084502\&uri= CELEX:32014R0269.

[9] Jia Deng, Wei Dong, Richard Socher, Li jia Li, Kai Li, and Li Fei-fei. 2009. Imagenet: A large-scale hierarchical image database. In In CVPR. CVPR, 8.

[10] Peter A. Flach. 2016. Classifier Calibration. Springer US, Boston, MA, 1-8. https://doi.org/10.1007/978-1-4899-7502-7_900-1

[11] Jerome Friedman, Trevor Hastie, and Robert Tibshirani. 2001. The elements of statistical learning. Vol. 1. Springer series in statistics New York.

[12] Yarin Gal and Zoubin Ghahramani. 2015. Dropout as a bayesian approximation Representing model uncertainty in deep learning. arXiv preprint arXiv:1506.02142 (2015), 12.

[13] Ian Goodfellow, Yoshua Bengio, and Aaron Courville. 2016. Deep Learning. MIT Press, New York, NY, USA. http://www.deeplearningbook.org.

[14] Chuan Guo, Geoff Pleiss, Yu Sun, and Kilian Q. Weinberger. 2017. On Calibration of Modern Neural Networks. In Proceedings of the 34th International Conference on Machine Learning - Volume 70 (Sydney, NSW, Australia) (ICML'17). JMLR.org, 1321-1330. http://dl.acm.org/citation.cfm?id=3305381.3305518

[15] T. Hastie, R. Tibshirani, and J.H. Friedman. 2009. The Elements of Statistical Learning: Data Mining, Inference, and Prediction. Springer. https://books.google. com/books?id=eBSgoAEACAAJ

[16] Geoffrey Hinton, Oriol Vinyals, and Jeff Dean. 2015. Distilling the knowledge in a neural network. arXiv preprint arXiv:1503.02531 (2015), 9.

[17] Andrew Ilyas, Shibani Santurkar, Dimitris Tsipras, Logan Engstrom, Brandon Tran, and Aleksander Madry. abs/1905.02175. Adversarial examples are not bugs, they are features. arXiv preprint arXiv:1905.02175 0, 0 (abs/1905.02175), 0.

[18] Ashish Kapoor, Kristen Grauman, Raquel Urtasun, and Trevor Darrell. 2007 Active learning with gaussian processes for object categorization. In 2007 IEEE 11th International Conference on Computer Vision. IEEE, IEEE, 1-8.

[19] Jinhan Kim, Robert Feldt, and Shin Yoo. 2019. Guiding Deep Learning System Testing Using Surprise Adequacy. In Proceedings of the 41st International Conference on Software Engineering (Montreal, Quebec, Canada) (ICSE '19). IEEE Press, Piscataway, NJ, USA, 1039-1049. https://doi.org/10.1109/ICSE.2019.00108

[20] Jakub Konečnỳ, H Brendan McMahan, Felix X Yu, Peter Richtárik, Ananda Theertha Suresh, and Dave Bacon. 2016. Federated learning: Strategies for improving communication efficiency. arXiv preprint arXiv:1610.05492 10 (2016).

[21] Alex Krizhevsky, Geoffrey Hinton, et al. 2009. Learning multiple layers of features from tiny images. Technical Report. Citeseer.

[22] Volodymyr Kuleshov, Nathan Fenner, and Stefano Ermon. 2018. Accurate uncertainties for deep learning using calibrated regression. arXiv preprint arXiv:1807.00263 (2018), 9

[23] Yann LeCun, Yoshua Bengio, and Geoffrey Hinton. 2015. Deep learning. Nature 521 (27 05 2015), 436 EP -. https://doi.org/10.1038/nature14539

[24] Yann LeCun, Léon Bottou, Yoshua Bengio, Patrick Haffner, et al. 1998. Gradientbased learning applied to document recognition. Proc. IEEE 86, 11 (1998), 22782324.

[25] Zenan Li, Xiaoxing Ma, Chang Xu, and Chun Cao. 2019. Structural Coverage Criteria for Neural Networks Could Be Misleading. In Proceedings of the 41st International Conference on Software Engineering: New Ideas and Emerging Results (Montreal, Quebec, Canada) (ICSE-NIER '19). IEEE Press, Piscataway, NJ, USA, 89-92. https://doi.org/10.1109/ICSE-NIER.2019.00031

[26] Zenan Li, Xiaoxing Ma, Chang Xu, Chun Cao, Jingwei Xu, and Jian Lu. 2019. Boosting Operational DNN Testing Efficiency through Conditioning. In Proceedings of the 27th ACM foint European Software Engineering Conference and Symposium on the Foundations of Software Engineering (ESEC/FSE '19). ACM, Tallinn, Estonia, 12. http://arxiv.org/abs/1906.02533

[27] Yun Liu, Krishna Gadepalli, Mohammad Norouzi, George E. Dahl, Timo Kohlberger, Aleksey Boyko, Subhashini Venugopalan, Aleksei Timofeev, Philip Q. Nelson, Greg S. Corrado, Jason D. Hipp, Lily Peng, and Martin C. Stumpe. 2017. Detecting Cancer Metastases on Gigapixel Pathology Images. arXiv:1703.02442 [cs.CV]

[28] L. Ma, F. Juefei-Xu, M. Xue, B. Li, L. Li, Y. Liu, and J. Zhao. 2019. DeepCT: Tomographic Combinatorial Testing for Deep Learning Systems. In 2019 IEEE 26th International Conference on Software Analysis, Evolution and Reengineering (SANER). ACM, New York, NY, USA, 614-618. https://doi.org/10.1109/SANER.
2019.8668044

[29] Lei Ma, Felix Juefei-Xu, Fuyuan Zhang, Jiyuan Sun, Minhui Xue, Bo Li, Chunyang Chen, Ting Su, Li Li, Yang Liu, Jianjun Zhao, and Yadong Wang. 2018. DeepGauge: Multi-granularity Testing Criteria for Deep Learning Systems. In Proceedings of the 33rd ACM/IEEE International Conference on Automated Software Engineering (Montpellier, France) (ASE 2018). ACM, New York, NY, USA, 120-131. https: //doi.org/10.1145/3238147.3238202

[30] Henning Mller, Paul Clough, Thomas Deselaers, and Barbara Caputo. 2010. ImageCLEF: Experimental Evaluation in Visual Information Retrieval (1st ed.). Springer Publishing Company, Incorporated.

[31] Emmanuel Montagnon, Milena Cerny, Alexandre Cadrin-Chênevert, Vincent Hamilton, Thomas Derennes, André Ilinca, Franck Vandenbroucke-Menu, Simon Turcotte, Samuel Kadoury, and An Tang. 2020. Deep learning workflow in radiology: a primer. Insights into Imaging 11, 1 (2020), 22. https://doi.org/10. 1186/s13244-019-0832-5

[32] Allan H. Murphy. 1973. A New Vector Partition of the Probability Score. Fournal of Applied Meteorology 12, 4 (1973), 595-600. https://doi.org/10.1175/15200450(1973)012<0595:ANVPOT>2.0.CO;2

[33] Mahdi Pakdaman Naeini, Gregory Cooper, and Milos Hauskrecht. 2015. Obtaining well calibrated probabilities using bayesian binning. In Twenty-Ninth $A A A I$ Conference on Artificial Intelligence. AAAI, 7.

[34] Andrew Ng. 2016. Nuts and bolts of building AI applications using Deep Learning. Neurips-Keynote (2016), 5.

[35] Alexandru Niculescu-Mizil and Rich Caruana. 2005. Predicting good probabilities with supervised learning. In Proceedings of the 22nd international conference on Machine learning. ACM, JMLR.org, 625-632.

[36] Ziad Obermeyer and Ezekiel J Emanuel. 2016. Predicting the future-big data, machine learning, and clinical medicine. The New England journal of medicine 375, 13 (2016), 1216

[37] Augustus Odena, Catherine Olsson, David Andersen, and Ian Goodfellow. 2019. TensorFuzz: Debugging Neural Networks with Coverage-Guided Fuzzing. In Proceedings of the 36th International Conference on Machine Learning (Proceedings of Machine Learning Research, Vol. 97), Kamalika Chaudhuri and Ruslan Salakhutdinov (Eds.). PMLR, Long Beach, California, USA, 4901-4911. http: //proceedings.mlr.press/v97/odena19a.html

[38] Sinno Jialin Pan and Qiang Yang. 2009. A survey on transfer learning. IEEE Transactions on knowledge and data engineering 22, 10 (2009), 1345-1359.

[39] Bo Pang, Lillian Lee, and Shivakumar Vaithyanathan. 2002. Thumbs Up? Sentiment Classification Using Machine Learning Techniques. In Proceedings of EMNLP. EMNLP, 79-86.

[40] Nicolas Papernot and Patrick McDaniel. 2018. Deep k-nearest neighbors: Towards confident, interpretable and robust deep learning. arXiv preprint arXiv:1803.04765 (2018), 18.

[41] Edoardo Pasolli and Farid Melgani. 2011. Gaussian process regression within an active learning scheme. In 2011 IEEE International Geoscience and Remote Sensing Symposium. IEEE, IEEE, 3574-3577.

[42] F. Pedregosa, G. Varoquaux, A. Gramfort, V. Michel, B. Thirion, O. Grisel, M. Blondel, P. Prettenhofer, R. Weiss, V. Dubourg, J. Vanderplas, A. Passos, D. Cournapeau, M. Brucher, M. Perrot, and E. Duchesnay. 2011. Scikit-learn: Machine Learning in Python. Journal of Machine Learning Research 12 (2011), 2825-2830.

[43] Kexin Pei, Yinzhi Cao, Junfeng Yang, and Suman Jana. 2017. DeepXplore: Automated Whitebox Testing of Deep Learning Systems. In Proceedings of the 26th Symposium on Operating Systems Principles (Shanghai, China) (SOSP '17). ACM, New York, NY, USA, 1-18. https://doi.org/10.1145/3132747.3132785

[44] Hung Viet Pham, Thibaud Lutellier, Weizhen Qi, and Lin Tan. 2019. CRADLE: cross-backend validation to detect and localize bugs in deep learning libraries. In Proceedings of the 41st International Conference on Software Engineering. IEEE Press, ICSE'19, 1027-1038.

[45] John C. Platt. 1999. Probabilistic Outputs for Support Vector Machines and Comparisons to Regularized Likelihood Methods. In ADVANCES IN LARGE MARGIN CLASSIFIERS. MIT Press, 61-74.

[46] Carl Edward Rasmussen and Christopher K. I. Williams. 2005. Gaussian Processes for Machine Learning (Adaptive Computation and Machine Learning). The MIT Press.

[47] Patrick Riley. 2019. Three pitfalls to avoid in machine learning. Nature 572, 7767 (Jul 2019), 27-29. https://doi.org/10.1038/d41586-019-02307-y

[48] Peter Schulam and Suchi Saria. 2019. Can you trust this prediction? Auditing pointwise reliability after learning. arXiv preprint arXiv:1901.00403 (2019), 10.

[49] Murat Sensoy, Lance Kaplan, and Melih Kandemir. 2018. Evidential deep learning to quantify classification uncertainty. In Advances in Neural Information Processing Systems. NeurIps, 3179-3189.

[50] Sambu Seo, Marko Wallat, Thore Graepel, and Klaus Obermayer. 2000. Gaussian process regression: Active data selection and test point rejection. In Mustererkennung 2000. Springer, 27-34.

[51] Burr Settles. 2009. Active learning literature survey. Technical Report. University of Wisconsin-Madison Department of Computer Sciences.

[52] Glenn Shafer and Vladimir Vovk. 2008. A tutorial on conformal prediction. fournal of Machine Learning Research 9, Mar (2008), 371-421. 
[53] Gabi Shalev, Yossi Adi, and Joseph Keshet. 2018. Out-of-distribution detection using multiple semantic label representations. In Advances in Neural Information Processing Systems. 7375-7385.

[54] Reza Shokri and Vitaly Shmatikov. 2015. Privacy-Preserving Deep Learning. In Proceedings of the 22Nd ACM SIGSAC Conference on Computer and Communications Security (Denver, Colorado, USA) (CCS '15). ACM, New York, NY, USA, 1310-1321. https://doi.org/10.1145/2810103.2813687

[55] Rui Shu, Hung Bui, Hirokazu Narui, and Stefano Ermon. 2018. A DIRT-T Ap proach to Unsupervised Domain Adaptation. In International Conference on Learning Representations. ICLR, 19. https://openreview.net/forum?id=H1q-TM-AW

[56] Youcheng Sun, Min Wu, Wenjie Ruan, Xiaowei Huang, Marta Kwiatkowska, and Daniel Kroening. 2018. Concolic Testing for Deep Neural Networks. In Proceedings of the 33rd ACM/IEEE International Conference on Automated Software Engineering (Montpellier, France) (ASE 2018). ACM, New York, NY, USA, 109-119. https://doi.org/10.1145/3238147.3238172

[57] Christian Szegedy, Vincent Vanhoucke, Sergey Ioffe, Jon Shlens, and Zbigniew Wojna. 2016. Rethinking the inception architecture for computer vision. In Proceedings of the IEEE conference on computer vision and pattern recognition. CVPR, 2818-2826.

[58] Ambuj Tewari and Peter L Bartlett. 2007. On the consistency of multiclass classification methods. Journal of Machine Learning Research 8, May (2007) 1007-1025.

[59] Michael Wainberg, Daniele Merico, Andrew Delong, and Brendan J Frey. 2018 Deep learning in biomedicine. Nature Biotechnology 36, 9 (2018), 829-838. https: //doi.org/10.1038/nbt.4233

[60] Huiyan Wang, Jingwei Xu, Chang Xu, Xiaoxing Ma, and Jian Lu. 2020. DIS SECTOR: Input Validation for Deep Learning Applications by Crossing-layer Dissection. In Proceedings of the 42st International Conference on Software Engineering. IEEE Press, ICSE'20, 12.

[61] Jindong Wang et al. 2019. Everything about Transfer Learning and Domain Adapation. http://transferlearning.xyz.

[62] Jingyi Wang, Guoliang Dong, Jun Sun, Xinyu Wang, and Peixin Zhang. 2019. Adversarial sample detection for deep neural network through model mutation testing. In Proceedings of the 41st International Conference on Software Engineering. IEEE Press, ICSE'19, 1245-1256.
[63] Xuezhi Wang, Tzu-Kuo Huang, and Jeff Schneider. 2014. Active Transfer Learning under Model Shift. In Proceedings of the 31st International Conference on Machine Learning (Proceedings of Machine Learning Research, 2), Eric P. Xing and Tony Jebara (Eds.). PMLR, Bejing, China, 1305-1313. http://proceedings.mlr.press/v32/ wangi14.html

[64] Bianca Zadrozny and Charles Elkan. 2001. Obtaining calibrated probability estimates from decision trees and naive Bayesian classifiers. Citeseer Citeseer, 7.

[65] Bianca Zadrozny and Charles Elkan. 2002. Transforming classifier scores into accurate multiclass probability estimates. In Proceedings of the eighth ACM SIGKDD international conference on Knowledge discovery and data mining. ACM, KDD, 694-699.

[66] John R. Zech, Marcus A. Badgeley, Manway Liu, Anthony B. Costa, Joseph J. Titano, and Eric Karl Oermann. 2018. Variable generalization performance of a deep learning model to detect pneumonia in chest radiographs: A cross-sectional study. PLOS Medicine 15, 11 (11 2018), 1-17. https://doi.org/10.1371/journal. pmed.1002683

[67] Jie M. Zhang, Mark Harman, Lei Ma, and Yang Liu. 2019. Machine Learning Testing: Survey, Landscapes and Horizons. CoRR abs/1906.10742 (2019), 35. arXiv:1906.10742 http://arxiv.org/abs/1906.10742

[68] Mengshi Zhang, Yuqun Zhang, Lingming Zhang, Cong Liu, and Sarfraz Khurshid. 2018. DeepRoad: GAN-based Metamorphic Testing and Input Validation Framework for Autonomous Driving Systems. In Proceedings of the 33rd ACM/IEEE International Conference on Automated Software Engineering (Montpellier, France) (ASE 2018). ACM, New York, NY, USA, 132-142. https://doi.org/10.1145/3238147. 3238187

[69] Zhi-Hua Zhou. 2016. Learnware: On the Future of Machine Learning. Front. Comput. Sci. 10, 4 (Aug. 2016), 589-590. https://doi.org/10.1007/s11704-016-69063

[70] Zhi-Hua Zhou. 2019. Abductive learning: Towards bridging machine learning and logical reasoning. Science China Information Sciences 62, 7 (2019), 76101.

[71] Jingbo Zhu, Huizhen Wang, Benjamin K Tsou, and Matthew Ma. 2009. Active learning with sampling by uncertainty and density for data annotations. IEE Transactions on audio, speech, and language processing 18, 6 (2009), 1323-1331.

[72] Xiaojin Jerry Zhu. 2005. Semi-supervised learning literature survey. Technical Report. University of Wisconsin-Madison Department of Computer Sciences. 